FORMATION Formation emploi

Revue française de sciences sociales

136 | octobre-décembre 2016

Pêle-mêle

\title{
Edito : Orientation, insertion : la construction des parcours, entre individuel et collectif
}

Jean-Frédéric Vergnies

\section{OpenEdition}

1 Journals

Édition électronique

URL : http://journals.openedition.org/formationemploi/4901

DOI : 10.4000/formationemploi.4901

ISSN : 2107-0946

Éditeur

La Documentation française

Édition imprimée

Date de publication : 31 décembre 2016

Pagination : 1-2

ISSN : 0759-6340

Référence électronique

Jean-Frédéric Vergnies, « Edito : Orientation, insertion : la construction des parcours, entre individuel et collectif », Formation emploi [En ligne], 136 | octobre-décembre 2016, mis en ligne le 31 décembre 2016, consulté le 30 octobre 2020. URL : http://journals.openedition.org/formationemploi/4901 ; DOI : https://doi.org/10.4000/formationemploi.4901

(c) Tous droits réservés 


\title{
Orientation, insertion : la construction des parcours, entre individuel et collectif
}

\author{
Jean-Frédéric Vergnies \\ Rédacteur en chef
}

Les parcours sociaux sont de plus en plus variés, surtout pour les jeunes. Ils sont tributaires de la multiplication des filières de formation (de la formation formelle à l'école aux formations en ligne ouvertes à tous), de la différenciation des régimes d'emploi (d'emploi à durée indéterminée jusqu’à intérimaire, voire auto-entrepreneur), des incertitudes économiques et du marché de l'emploi...

Comment se constituent les parcours dans un tel contexte évolutif ? À travers quelques exemples dans différents pays, ce numéro invite à considérer les parcours individuels comme encastrés dans des dimensions collectives.

Ainsi, en Suisse, la politique éducative intègre depuis longtemps l'employabilité des jeunes. Geneviève Mottet analyse ici la genèse et l'essor d'une politique sociale active dans le domaine de l'éducation.

Pourtant, les histoires et les choix individuels ne rencontrent pas toujours cette volonté institutionnelle de modéliser les parcours. De fait, les jeunes qui aspirent à suivre une formation en apprentissage sont loin d'avoir le comportement attendu. Comme le montre Marie-Hélène Jacques, certains vont poursuivre leurs études alors qu'ils suivaient une formation censée déboucher directement sur le marché de l'emploi, tandis que d'autres se réorientent, voire arrêtent leurs études pour chercher un emploi.

Ainsi, la socialisation primaire, se construisant durant l'enfance, est déjà marquée par la diversité sociale des familles et la diversité institutionnelle de l'École.

La formation tout au long de la vie est encore plus marquée par la différenciation des parcours. Un diplôme comme celui d'entraîneur de football professionnel ouvre sur des emplois différents selon les avantages cumulés lors des parcours sportifs et professionnels antérieurs, comme le précisent Jean Bréhon, Hugo Juskowiak \& Loïc Sallé. 
Au-delà des caractéristiques individuelles identifiables (origine sociale, formation, emplois occupés...), ces parcours sont aussi conditionnés par l'environnement de travail, comme le rappellent Justine Arnoud \& Cathel Kornig. Ainsi, pour les professionnels de l'enseignement supérieur et de la recherche, la qualité du travail (performance, santé...) dépend largement des ressources psychosociales mobilisables (notamment l'autonomie, le collectif de travail, la reconnaissance des pairs et de la hiérarchie, la formation, les outils de travail et les nouvelles formes d'organisation liées aux évolutions institutionnelles...).

Cette socialisation secondaire reste cependant parfois incomplète, et les institutions tentent aussi d'y remédier.

Ainsi, en Belgique, Julie Gérard \& Didier Vrancken montrent comment les dispositifs visant à réinsérer les demandeurs d'emploi peu qualifiés aboutissent, en définitive, plutôt à les acclimater à l'incertitude et aux aléas des parcours de vie et d'emploi.

En France, les entreprises attributaires de marchés publics ont l'obligation de proposer un nombre minimal d'heures de travail à des personnes éloignées de l'emploi. Une partie des bénéficiaires des clauses d'insertion des marchés publics parviennent alors à sécuriser leurs parcours professionnels. Selon Nathalie Havet, Alexis Penot \& Morgane Plantier, le maintien en emploi dépend de la capacité de ce dispositif à améliorer la confiance en soi des bénéficiaires et à leur faire acquérir ... une formation. Affaire à suivre.

Bonne lecture. 